\title{
Allee optimal control of a system in ecology
}

\author{
Emmanuel Trélat * $\quad$ Jiamin Zhu ${ }^{\dagger} \quad$ Enrique Zuazua $¥ \S \uparrow \|$
}

\begin{abstract}
The Allee threshold of an ecological system distinguishes the sign of population growth either towards extinction or to carrying capacity. In practice human interventions can tune the Allee threshold for instance thanks to the sterile male technique and the mating disruption. In this paper we address various control objectives for a system described by a diffusion-reaction equation regulating the Allee threshold, viewed as a real parameter determining the unstable equilibrium of the bistable nonlinear reaction term.

We prove that this system is the mean field limit of an interacting system of particles in which individual behaviours are driven by stochastic laws. Numerical simulations of the stochastic process show that population propagations are governed by wave-like solutions corresponding to traveling solutions of the macroscopic reaction-diffusion system.

An optimal control problem for the macroscopic model is then introduced with the objective of steering the system to a target traveling wave. The relevance of this problem is motivated by the fact that traveling wave solutions model the fact that bounded space domains reach asymptotically an equilibrium configuration. Using well known analytical results and stability properties of traveling waves, we show that well-chosen piecewise constant controls allow to reach the target approximately in sufficiently long time. We then develop a direct computational method and show its efficiency for computing such controls in various numerical simulations. Finally we show the efficiency of the obtained macroscopic optimal controls in the microscopic system of interacting particles and we discuss their advantage when addressing situations that are out of reach for the analytical methods.

We conclude the article with some open problems and directions for future research.
\end{abstract}

Keywords: diffusion-reaction equation, interacting particle system, stochastic process, traveling wave, optimal control, ecological system, Allee effect, piecewise control strategy, direct computational method.

\section{Introduction, motivation and problem formulation}

Macroscopic model and Allee effect. We consider the reaction-diffusion equation

$$
\partial_{t} y(t, x)-\partial_{x x} y(t, x)=f(y(t, x), \theta), \quad x \in \mathbb{R}, \quad t \in[0,+\infty)
$$

where

$$
f(y, \theta)=r y(1-y)(y-\theta)
$$

\footnotetext{
* Sorbonne Université, Université Paris-Diderot SPC, CNRS, Inria, Laboratoire Jacques-Louis Lions, équipe CAGE, F-75005 Paris (emmanuel.trelat@sorbonne-universite.fr)

†Université Paul Sabatier, Institut de Mathématiques de Toulouse, UMR5219, F-31062 Toulouse (zhu@ann.jussieu.fr)

${ }^{\ddagger}$ DeustoTech, University of Deusto, 48007 Bilbao, Basque Country, Spain (enrique.zuazua@deusto.es)

$\S$ Departamento de Matemáticas, Universidad Autónoma de Madrid, 28049 Madrid, Spain

『Facultad de Ingeniería, Universidad de Deusto, Avda. Universidades, 24, 48007 Bilbao, Basque Country, Spain

"Sorbonne Université, Université Paris-Diderot SPC, CNRS, Laboratoire Jacques-Louis Lions, F-75005 Paris
} 
is the classical bistable reaction term describing the population change from birth and death and $y:[0,+\infty) \times \mathbb{R} \rightarrow \mathbb{R}$ is the local population density.

System (1) is a typical model in ecology modelling the propagation of a population of organisms (see $[44,50]$ ). The growth of the population, in addition to migration, which is described by the diffusion term $\partial_{x x} y$, is subject to an Allee effect that is modelled through the nonlinearity.

The parameter $r>0$, assumed to be fixed in our analysis, represents the reproduction rate and can be normalised to $r=1$ through a change of scale in space and time. The parameter $\theta \in(0,1)$ is the local critical density and is called Allee threshold; it determines the sign (positive or negative) of the population growth (see Fig. 1(left)). The Allee effect, modelled by this reaction term, expresses the fact that the population growth is negative when the density of the population is lower than the Allee threshold $\theta$, while, otherwise, when the density of the population is higher than this threshold, the population tends to reach the carrying capacity.

Steady-states and traveling waves. System (1) has three constant (independent of the space variable $x$ ) solutions: $y \equiv 0$ (stable, extinction), $y \equiv \theta$ (unstable), and $y \equiv 1$ (stable, carrying capacity). When $y$ tends to 0 , we say that the population extinguishes, and when $y$ tends to 1 , we way that population invades.

Of course the set of steady-state solutions is much richer as can be seen by a phase plane analysis. But our analysis here will be mainly devoted to these constant steady-states since they represent the simplest stable configurations in which all population reaches the same value, uniformly, independent of the space-variable $x$.

The system exhibits also traveling wave solutions of the form

$$
y(t, x)=Y(x-c t)
$$

linking two steady-states (most often the stable ones 0 and 1) with an invariant wave profile $Y$ and a constant wave speed $c$ (see Fig. 1(right)). Of course, these solutions, depending only on one space variable $s=x-c t$, can also be viewed as planar wave solutions in multi-D.
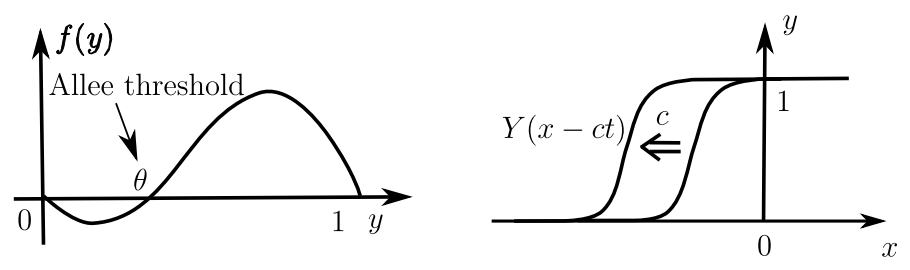

Figure 1: Reaction term $f(y)$ (left); traveling wave (right).

Motivation of the model. This kind of model appears in many applications in chemistry, mathematical biology and social sciences. It has been used to describe the propagation of an allele in a population (see $[4,34]$ ), to simulate the propagation of a voltage pulse through an animal nerve axon (see [41, 55]) and to describe the propagation of chemical steady-states in chemical reactions in a continuous stirred tank reactor (see [30,49]). We refer to [54, 59] for applications to biology. The model is also relevant in many applications in social behaviour studies. It may be viewed as a diffusive version of the game theoretical and ODE models introduced in [42] for minority languages.

Traveling wave solutions linking the two stable equilibria play an essential role in the dynamical behaviour of these systems since they describe how, as time evolves, space domains that are occupied by the configuration $y \sim 1$ tend towards the other stable value $y \sim 0$ or vice-versa. Complementing [42] this phenomenon provides a simple explanation of how the interplay between diffusion and nonlinearity contributes to the contraction of minority languages. 
System (1) is a macroscopic (or phenomenological) model for a group of living individuals, in contrast to microscopic models for individual behaviours. In Section 2 we prove that (1) is the mean field limit of a stochastic system of interacting particles in which the individuals of the population are considered as active particles that behave according to some probabilistic laws. Numerical simulations of this stochastic system show a wave-like propagation pattern corresponding to traveling wave solutions of (1).

The Allee threshold as a control. The latter fact provides a second motivation to consider optimal control problems for system (1) with the objective of driving it to an expected traveling wave, the Allee threshold parameter $\theta$ being the control parameter. The choice of the parameter $\theta$ as a control, making it time-dependent, is a natural one as a mean to use the Allee effect in order to realize control objectives.

In order to understand the role that $\theta$ may play as an actuator, it is useful to exploit the stability and asymptotic properties of the system for a given fixed value of $\theta$. For example, when the value of $0<\theta<1$ is time-independent, it is well known that extinction of population occurs if the initial density is (uniformly in $x$ ) smaller than $\theta$ (it may even be larger than $\theta$ but only on a small enough domain). It is also well known that population invasion occurs if the initial density is larger than $\theta$ on a sufficiently large domain (see [4, 32]). Therefore, for a fixed initial datum, choosing the appropriate value of a time-independent $\theta$, one may change an invasion (or extinction) dynamics into an extinction (or invasion) one. Of course, the time needed for these phenomena to occur also depends on the specific value of the parameter $\theta$. This is the first manifestation of the role that $\theta$ may play as a control parameter.

The use of the dynamically changing value of the Allee parameter $\theta=\theta(t)$ as a control or actuator to modify the dynamics of the system is fully justified from a practical and applied viewpoint as well. For instance, in [51], the authors refer to several ecological measures to control the spread of the population by taking advantage of the Allee effect. On the one hand, the sterile male technique and the mating disruption can be used to increase the Allee threshold $\theta$. The former has been successfully used to control the spread of the invading insect species such as the Mediterranean fruit fly, the melon fly (Bactrocera cucurbitae), and the Mexican fruit fly (Anastrepha ludens) (see [47]), and the latter has been used in the gypsy moth containment program. On the other hand, the Allee threshold can be decreased by providing protection (like efficient feeding, suppressing natural enemies) to the population.

Another method that has shown efficiency in the control of mosquito populations is the addition of clusters of mosquitoes infected by Wolbachia, modeled with the aid of the Allee effect (see [8, 67]). In [16] a scalar bistable reaction-diffusion model is derived and stabilised by means of a distributed additive feedback law with compact support. This paper describes how the added and localised population of infected mosquitoes may invade the whole space by taking advantage of the Allee threshold effect. The control we employ in the present paper is however more subtle since it enters through the Allee parameter and not as an additive source perturbing the dynamics.

The role that the Allee parameter plays as an actuator can also be justified from a microperspective. In Section 2.3 we describe how the value of $\theta$ can be tuned by a more complex interacting particle system describing the population evolution under the action of an applied sterile male technique.

Driving the system to a traveling wave. Our goal is to mathematically and computationally analyse these evidences that the dynamical shaping of the time-dependent Allee threshold $\theta=\theta(t)$ can lead to satisfactory control performances. We formulate the problem from the point of view of optimal control, aiming to design the optimal actuator strategy $\theta=\theta(t)$ in such a way that the evolution of the state tracks an a priori given target trajectory. We focus on the particular case where the target is a traveling wave solution. This is motivated by the fact that traveling waves describe a dynamics in which the asymptotic behaviour in long time is known a priori. Being able to drive the state close to some traveling wave solutions, thanks to their well known stability 
properties, we therefore guarantee that, locally in space, the state is driven close to the asymptotic value of the traveling wave and more precisely to the stable steady-states attracting locally its dynamics.

We prove a result of approximate controllability in large time showing that the solutions (for a wide class of initial data) can be driven arbitrarily close to the traveling wave target if time is large enough. This result is established using well known stability properties of traveling waves. It is not addressed by means of the classical tools of controllability theory for parabolic equations for two main reasons: the first one is that the control $\theta$ enters on the system as a coefficient in the nonlinearity; the second one is that the control is only a scalar function $\theta(t)$ depending on time and is not an applied force distributed in space as in most of the existing literature (see [74]). However the precise understanding of the dynamical behaviour of the system allows building piecewise constant control strategies ensuring approximate controllability in large time.

To be more precise, the optimal control problem that we address consists of designing a control law $\theta(t), t \in[0, T]$, such that the population density $y$ approaches a desired distribution $y_{d}(x)$ (corresponding to a traveling wave profile) at a given time $T$ in a bounded domain $\Omega \subset \mathbb{R}$. Our analytical results, however, need a control time $T=T(\varepsilon)$ depending on the admissible distance $\varepsilon$ to the target.

One of the main differences of our work with respect to classical boundary or distributed control (external forcing) problems (see [7, 24, 25, 65, 70]) is that we control the system by the Allee parameter. The literature on control of PDEs when the control is a scalar parameter entering in the system in a nonlinear way is rather limited. Significant results on this topic concern bilinear controllability problems for Schrödinger, heat, and more general parabolic equations and can be found in $[9,19,23,31]$ (see also references therein).

The approximate controllability result in large time above is limited in scope since, in practical applications, the time horizon $T$ for control is given a priori. It is therefore natural to consider the problem from the point of view of optimisation. We also formulate the problem with this viewpoint and we propose a direct computational method consisting of performing a minimisation procedure on a discrete version of the problem where the state equation and the cost functional have been discretised. The resulting minimisation problem is not convex and the minimiser fails to be unique in general. The numerical controls that we find thus depend on the initialisation. As we shall see, interestingly this method is however capable of recovering the piecewise control strategy that we developed analytically.

Once the optimal control strategy has been found for the macroscopic model, either analytically or computationally, the question arises of whether these controls are also efficient to control the microscopic model and observe the desired "mean" behaviour of all individuals in the population. Our numerical simulations confirm the adequacy of the control strategies at this level too.

Organization of the paper. The paper is structured as follows:

- In Section 2, we model and construct a system of particles interacting with probabilistic laws and we show that its mean field limit is the macroscopic model (1). We provide numerical evidence that the propagation of population for this microscopic system is naturally described by wave-like solutions such as traveling waves. We introduce a more complex interacting particle system (modelling population dynamics under sterile male techniques) to show that the control parameter $\theta$ can indeed be tuned and manipulated.

- In Section 3, after having recalled some classical results on traveling wave solutions, we formulate two optimal control problems. The first one, denoted by $(\mathcal{P})_{\text {opt }}$, concerns system (1) and consists of minimising the distance between the state of the system and an expected "target" traveling solution. The second one, denoted by $(\mathcal{P})_{S I R M}$, concerns the extended system with sterile male techniques, aiming at eliminating the pest population by using the least possible amount of sterile insects. This extended problem justifies the feasibility of controlling the system by means of tuning adequately in time the Allee threshold. 
- In Section 4, we establish approximate controllability results in large time for $(\mathcal{P})_{\text {opt }}$.

- In Section 5, we present numerical simulations for $(\mathcal{P})_{\text {opt }}$ and $(\mathcal{P})_{\text {SIRM }}$.

- In Section 6 we provide numerical evidence that the deterministic control strategies obtained for the macroscopic model still work when applied to the microscopic system of interacting particles constructed in Section 2.

- In Section 7, we give some concluding remarks and propose some open problems.

\section{The microscopic model}

We show in this section that the macroscopic system (1) represents in some sense the dynamics of a microscopic system for individual behaviors.

\subsection{System of interacting particles}

A system of interacting particles is a stochastic process with state space $E^{\mathbb{Z}^{d}}$, where $E$ is some finite or countably infinite set and $\mathbb{Z}^{d}$ is the integer space of dimension $d$. We call $x \in \mathbb{Z}^{d}$ a site, allowed to take values in the set of states $E$. By properly choosing the stochastic process, the reaction-diffusion limit is obtained by scaling space and introducing fast stirring so that $\mathbb{Z}^{d}$ is scaled to $\varepsilon \mathbb{Z}^{d}$ and the exchange between neighbouring sites occurs at rate $1 / \varepsilon^{2}$.

As shown in [53], the macroscopic density viewed on a spatial scale of order $\varepsilon^{-1}$ evolves according to an autonomous nonlinear diffusion-reaction equation (1) with the reaction term $f(y)$ depending on the chosen process. For example the logistic reaction $f(y)=y-y^{2}$ follows from the so-called contact process (see $[27,28,56,57]$ ). This process essentially assumes that individuals die at a rate that is independent of the state of their neighbours, while births at vacant sites occur at a rate proportional to the number of occupied neighbours. More complicated models such as the classical prey-predator model and the chemotaxis model can also be derived in this way from stochastic models (see [27, 64, 73]).

Intuitively a site $x$ is like a "house" and a particle can be seen as an animal individual. A house is either occupied or vacant meaning that there may be an animal living inside or not. The stochastic process describes how these animals reproduce and die according to their environment and exchanges occurring at a fast rate (of the order of $1 / \varepsilon^{2}$ with $\varepsilon$ small) between neighbouring sites. This expresses that the motion of the animal individuals (visiting their neighbours) is more frequent than their reproduction or dead rate.

Let us next construct this stochastic process in more detail in order to ultimately obtain the model (1) by mean field limit.

We proceed as in [27]. Let $\varepsilon>0$ be the scale parameter, let $\varepsilon \mathbb{Z}^{d}$ be the set of spatial locations and let $E=\{0,1\}$ be the set of states. Each site of $\varepsilon \mathbb{Z}$ can be either vacant (i.e., at state 0 ), or occupied (i.e., at state 1 ). The system is then described at time $t$ by the configuration $\zeta_{t} \in\{0,1\} \mathbb{Z}^{d}$.

We say that $x_{1}$ and $x_{2} \in \varepsilon \mathbb{Z}^{d}$ are neighbors if $\left\|x_{1}-x_{2}\right\|=\varepsilon$. Here and in the sequel $\|\cdot\|$ stands for the Euclidean norm. We denote by $n_{i}(x, \zeta)$ the number of neighbours of site $x$ at state $i \in E$. We assume that with the given configuration $\zeta$, the site $x \in \varepsilon \mathbb{Z}$ flips to state $i$ at rate $c_{i}(x, \zeta)$, with

$$
\begin{cases}c_{1}(x, \zeta)=r \frac{n_{1}\left(n_{1}-1\right)}{N(N-1)}, & \text { if } \zeta(x)=0, n_{1}(x, \zeta) \geqslant 2, \\ c_{0}(x, \zeta)=r \frac{\theta}{N} n_{0}(x, \zeta), & \text { if } \zeta(x)=1,\end{cases}
$$

where $N$ is the number of neighbors of a site. For example, when $d=2($ resp., $d=1$ ), each site has four neighbors, i.e., $N=4$ (resp., $N=2$ ). In this stochastic process : 
- $c_{1}$, the birth rate of an individual, is proportional to the number of neighbours occupied. For a new birth to occur there must be at least two neighbors occupied. When the neighbors are all occupied then at a maximum rate $r$ there will be a new birth to occupy the site $x$.

- $c_{0}$, the death rate, is proportional to $n_{0}(x, \zeta)$. The more empty neighbours $x$ has, the higher the rate of death is (becoming 0 ) at the site $x$. When the neighbours are all empty then at a maximum rate $r \theta$ the individual at site $x$ will die.

These rules are consistent with the macroscopic Allee phenomenon: when an individual lives with enough other individuals it has more chances to reproduce; otherwise it has more chances to die. This actually happens to certain (especially social) animals.

Taking into account that the exchange between neighbouring sites is at rate of the order of $1 / \varepsilon^{2}$ (fast stirring) and according to [27, Theorem 8.1] (based on the mean field limit theorem of [53]), we obtain that the limiting equation is (1).

Theorem 1. Assume that the processes $\zeta_{0}^{\varepsilon}(x)\left(x \in \mathbb{Z}^{d}\right)$ are independent. Let $y^{\varepsilon}(t, x)=P\left(\zeta_{t}(x)=\right.$ 1) be the probability for $\zeta_{t}$ to be of state 1 at site $x$. Assume that $g(x)=\lim _{\varepsilon \rightarrow 0} y^{\varepsilon}(0, x)$ is continuous. Then $y^{\varepsilon}(t, x)$ converges as $\varepsilon \rightarrow 0$ to the bounded solution $y(t, x)$ of (1) with initial datum $y(0, x)=g(x)$.

Another important approach to derive complex macroscopic models with diffusion from microscopic ones is to start from the kinetic transport (Boltzmann-type) equations (see [10, 13, 15, 18, $40,58]$ ). In biology, this approach is especially useful for describing the motion of numerous individuals in chemotaxis (see [22, 33]), crowd dynamics (see [11, 26]), opinion modeling (see [1, 6]). In our model (1), we do not consider crowd motions and thus we will not discuss further this method. However, the kinetic transport approach can surely help when one expects to deal with populations who can make decisions on their moving behaviour depending on environmental factors. This is a subject that would deserves further study in the context of modelling multilingualism (see [42]).

\section{$2.2 \quad$ Numerical simulations}

We use a Markov method (see [73]) to simulate the evolution of the population distribution according to the stochastic process (2). We observe that wave-like solutions emerge: traveling waves in $1 \mathrm{D}$ and radial waves in $2 \mathrm{D}$.
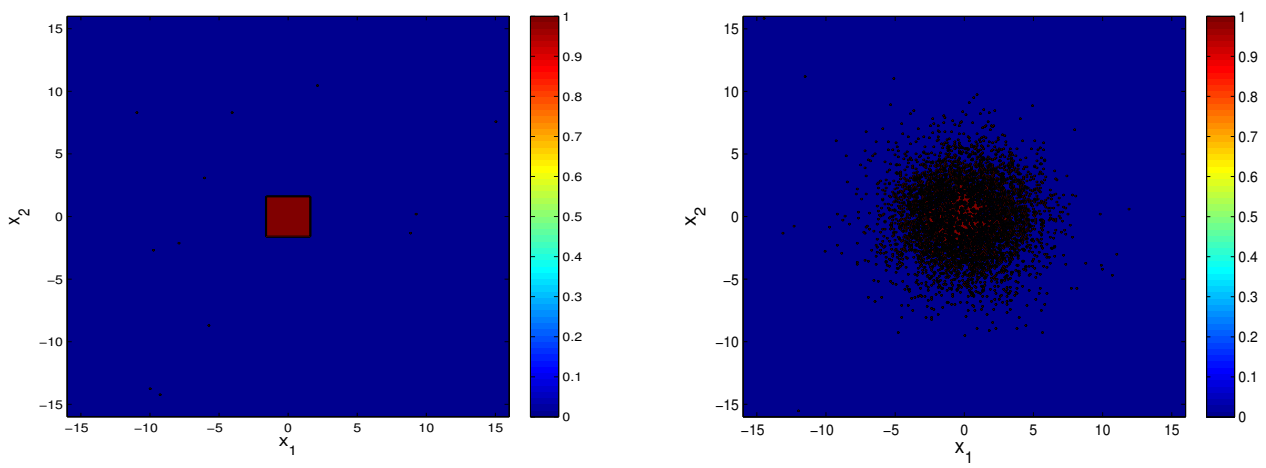

Figure 2: Evolution of the number of the population individuals: initial configuration (left); configuration at time $t=15$ (right).

When $d=2$ (i.e., $x \in \mathbb{Z}^{2}$ ), the obtained population distribution is given in Fig. 2. Here we have set $r=1, \theta=0.1$, with initial configuration on a subset $[-200,200]^{2}$ of $\mathbb{Z}^{2}$ as shown in Fig. 
2 (left) with $\varepsilon=0.08$. Note that $[-200,200]^{2}$ means that there are $400^{2}$ "houses" and thus the same maximum number of individuals. We can see in Fig. 2 (right) that the number of individuals increases with time and the observed propagation is somehow symmetric and wave-like.

The stochastic model is closer to the intrinsic complexity of the population dynamics but a high computational effort is required, difficult to be parallelised (see [27, 26]). Thus a macroscopic model (also referred to as phenomenological) describing the behavior of the average density of the population is generally preferable to address analytical and optimization or control issues (see $[12,20,38,72])$.

In the next example, we show that the macroscopic model provides a good approximation of the complex stochastic dynamics. Let $d=1$ (i.e., $x \in \mathbb{Z}$ ), $\mathbb{Z}=[-500,500]$, and $\varepsilon=0.05$ and we run the simulation $M=100$ times. We take the "mean" population distribution $\bar{y}(T, x)=\frac{1}{M} \sum_{1}^{M} y^{i}(T, x)$, where $y^{i}$ is the distribution obtained during the $i$-th simulation. A comparison between this mean solution and the solution of the macroscopic model (1) is given in Fig. 3. The red and green lines represent mean population distributions of model (2) at initial time 0 and at final time $T$, while the green and blue lines are computed according to the explicit traveling wave solution (6) and (7) for the macroscopic model (1) (see next section). This simulation shows that the averaged behaviour of living individuals (particles) is consistent with the solution of the deterministic model (see Fig. 2).

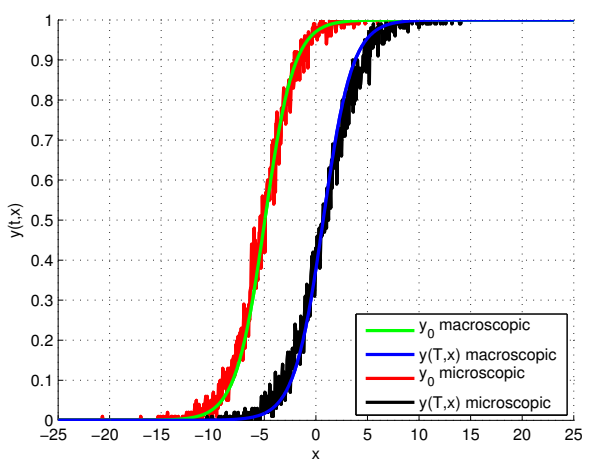

Figure 3: Comparison of solutions obtained from macroscopic and stochastic (microscopic) models.

\subsection{Extension: a stochastic sterile male model}

We briefly present in this section a stochastic sterile male model showing that the Allee threshold parameter $\theta$ can be modified and tuned to play the role of a control/actuator.

As mentioned in the introduction, the Allee threshold $\theta$ can be modified by human interaction, such as sterile insect release method (SIRM). This justifies to consider it as a control parameter. The SIRM is known to be non-pulling and effective in many situations but rather expensive due to the need of feeding numerous sterile males (see [51, 43, 48, 68] for other models and applications of SIRM). In this section we construct a more complex microscopic model that describes the population evolution while considering the effect of the SIRM. We will see that the Allee threshold $\theta$ can actually be modified by a second equation.

Let us first introduce the corresponding averaged macroscopic model. We do it in 2D although a similar construction can be done in $1 \mathrm{D}$ as well. Let $y_{1}(t, \mathbf{x}) \in \mathbb{R}$ and $y_{2}(t, \mathbf{x}) \in \mathbb{R}$ be respectively densities of normal and sterile couples (a female and a male) at time $t \in \mathbb{R}_{+}$at position $\mathbf{x}=$ $\left(x_{1}, x_{2}\right)^{\top} \in \mathbb{R}^{2}, r>0$ be the birth rate of $y_{1}$, let $\theta \in(0,1)$ be the Allee threshold of $y_{1}$, and let $u>0$ is the transformation rate from $y_{1}$ to $y_{2}$. Note that the released sterile males "steal" 
the females in normal couples and thus transforms a normal couple into a sterile one. Denoting $\mathbf{y}=\left(y_{1}, y_{2}\right)^{\top}$, the macroscopic model is

$$
\frac{\partial}{\partial t} \mathbf{y}-\triangle \mathbf{y}=F(\mathbf{y})
$$

with reaction term $F=\left(f_{1}, f_{2}\right)^{\top}$ with $f_{1}=r y_{1}(1-y)(y-\theta)-u y_{1}$ and $f_{2}=u y_{1}-r \theta y_{2}(1-y)$ where $y=y_{1}+y_{2}$ is the total population density. Note that the total population density $y$ satisfies

$$
\frac{\partial}{\partial t} y-\Delta y=r y(1-y)\left(y-\left(\theta+y_{2}\right)\right)
$$

which indicates that the density of sterile population $y_{2}$ can increase the Allee threshold $\left(\theta+y_{2}\right)$ for the total insect density $y$.

Using the method explained in Section 2.1, a microscopic model leading to (1) can be built as well as follows. Let $\varepsilon \mathbb{Z}^{d}$ be the set of spatial locations and $\{0,1,2\}$ be the set of states: 0 means that the site is vacant; 1 means that the site is occupied by a normal couple; 2 means that the site is occupied by a sterile couple. Each site of $\varepsilon \mathbb{Z}^{d}$ can be at these three possible states, 0 , or 1 , or 2. The system is described at time $t$ by the configuration $\zeta_{t} \in\{0,1,2\}^{\varepsilon \mathbb{Z}^{d}}$. Recall that $\mathbf{x}_{1}$ and $\mathbf{x}_{2} \in \varepsilon \mathbb{Z}^{d}$ are neighbours if $\left\|\mathbf{x}_{1}-\mathbf{x}_{2}\right\|=\varepsilon$, and $n_{i}(\mathbf{x}, \zeta)$ is the number of neighbours of site $\mathbf{x}$ at state $i \in\{0,1,2\}$. We assume that with the given configuration $\zeta$, site $\mathbf{x} \in \varepsilon \mathbb{Z}^{d}$ flips to state $i$ at rate $c_{i}(\mathbf{x}, \zeta)$ with

$$
\begin{aligned}
& c_{1}(\mathbf{x}, \zeta)=r \frac{n_{1}\left(n_{1}-1\right)+n_{1} n_{2}}{N(N-1)}, \quad \text { if } \zeta(\mathbf{x})=0, n_{1}(\mathbf{x}, \zeta) \geqslant 1, n_{1}(\mathbf{x}, \zeta)+n_{2}(\mathbf{x}, \zeta) \geqslant 2, \\
& c_{2}(\mathbf{x}, \zeta)=u, \quad \text { if } \zeta(\mathbf{x})=1, \\
& c_{0}(\mathbf{x}, \zeta)= \begin{cases}r \frac{\theta}{N} n_{0}(\mathbf{x}, \zeta)+u & \text { if } \zeta(\mathbf{x})=1, \\
r \frac{\theta}{N} n_{0}(\mathbf{x}, \zeta) & \text { if } \zeta(\mathbf{x})=2,\end{cases}
\end{aligned}
$$

where $N$ is the number of neighbours of a site. The diffusion term comes from the fast exchange between neighbouring sites and the reaction one from the reproduction and death given by (5). In the stochastic model (5):

- $c_{1}$, the birth rate of the normal couple, is proportional to the number of neighbors occupied. For having a new birth there must be at least two neighbours occupied (normal or sterile) and there must be at least one normal couple in the neighbours. When the neighbours are all occupied by normal individuals, there will be a new birth to occupy the site $\mathbf{x}$ at a maximum rate $r$.

- $c_{2}$ is the "birth" rate of the sterile couple: at rate $u$, a normal couple transforms to a sterile one. When sterile males are related in the field they would be able to "steal" with some probability the females of normal couples and thus transform normal couples to sterile ones. Moreover, $c_{2}$ also indicates that the sterile males are introduced only when there are still normal ones.

- $c_{0}$ is the death rate of the insects. Note that the death rates of normal and sterile couples are the same except for the additive term $u$. The term proportional to $n_{0}(\mathbf{x}, \zeta)$ means that the more empty neighbours $\mathbf{x}$ has, the higher is the rate of death.

The rules describing (2) are consistent with the Allee effect: when a normal couple lives there with sufficient other ones, it has more chances to reproduce, otherwise, it has more chances to die. When the neighbours are all empty, then at a maximum rate $r \theta$, the insect at site $\mathbf{x}$ will die.

According to [27, Theorem 8.1] the limiting equation is (3). 
Theorem 2. Assume that the processes $\zeta_{0}^{\varepsilon}(x)\left(x \in \mathbb{Z}^{d}\right)$ are independent. Let $y_{i}^{\varepsilon}(t, x)=P\left(\zeta_{t}(x)=\right.$ i) for $i=1,2$ be the probability for $\zeta_{t}$ to be of state $i$ at site $x$. Assume that $g_{i}(x)=\lim _{\varepsilon \rightarrow 0} y_{i}^{\varepsilon}(0, x)$ is continuous. Then $y_{i}^{\varepsilon}(t, x)$ converges as $\varepsilon \rightarrow 0$ to the bounded solution $y_{i}(t, x)$ of (3) such that $y_{i}(0, x)=g_{i}(x), i=1,2$.

System (4) is an example in which the Allee threshold $\theta$ (the control parameter for the macroscopic model (1)) can be tuned. As in Section 2.2, we observe on numerical simulations the occurrence of wave solutions (radial waves in $2 \mathrm{D}$ and traveling waves in 1D).

The rest of this paper is devoted to the control of the macroscopic model (1) in 1D. We will then apply the controls obtained in the macroscopic model (1) to the stochastic process to show its good performance. Although the techniques of this paper could also be applied in multi-D, the 1D case allows for a more precise comparison between microscopic and macroscopic models. Before addressing the control problems, in the next section we recall some basic results on traveling waves.

\section{Macroscopic optimal control problems}

\subsection{Several reminders on traveling waves}

There is an extensive literature on well-posedness and asymptotic behaviour of traveling waves (see [3, 4, 32]). Concerning the well-posedness of (1), it is well known that if the initial data $y_{0}$ is piecewise continuous and if $0 \leqslant y_{0}(x) \leqslant 1$ for all $x \in \mathbb{R}$ then there exists a unique bounded classical solution $y(t, x)$ of $(1)$, satisfying $0 \leqslant y(t, x) \leqslant 1$ for all $(t, x) \in[0,+\infty) \times \mathbb{R}$. For the system (1) with $r>0$ and $\theta \in(0,1)$, the steady-states $y=0$ and $y=1$ are stable while the steady-state $y=\theta$ is unstable. Any traveling wave solution of (1) takes the form $y(t, x)=Y(x-c t$ ) where $c$ (wave speed) is a real number and $Y$ is the wave profile. We denote by $s=x-c t$ the moving coordinate. Without loss of generality, we focus on waves satisfying the auxiliary conditions $Y(s) \in[0,1]$, $Y(s) \not \equiv 0$, and

$$
Y(s) \stackrel{s \rightarrow-\infty}{\longrightarrow} y_{-}, \quad Y(s) \stackrel{s \rightarrow+\infty}{\longrightarrow} y_{+},
$$

where $y_{-}$and $y_{+}$are finite limits taking values in three constant solutions of (1), i.e., $0, \theta$ and 1 that are the three roots of the reaction term $f$. To fix ideas we set $y_{-}=0$ and $y_{+}=1$. Similarly, replacing $x$ by $-x$, one could also consider the reverse situation $y_{-}=1$ and $y_{+}=0$.

The unstable steady-state $\theta$ is not considered here as one of the possible asymptotic state of the traveling wave profiles since the system behaviour of a stochastic model around an unstable point is not reproduced by the macroscopic model. Explicit traveling wave solutions of system (1) are known (see [46, pp. 270-274]) and are given by the profile

$$
y(x-c t)=Y(s)=\frac{e^{-\sqrt{r / 2}\left(s-s_{0}\right)}}{1+e^{-\sqrt{r / 2}\left(s-s_{0}\right)}}
$$

where $s_{0}$ is the constant determining the initial position (at $t=0$ ) of the traveling wave. It is important to observe that the profile $Y$ of the traveling wave is independent of the value of $\theta$ while its velocity of propagation is given by

$$
c(\theta)=\sqrt{r / 2}(1-2 \theta) .
$$

The sign of $c(\theta)$ and, accordingly, the direction of propagation of the traveling front, depends on whether $\theta<1 / 2$ (resp. $\theta>1 / 2$ ), in which case $c>0$ (resp., $c<0$ ). When $\theta \rightarrow 1 / 2$ the velocity $c(\theta) \rightarrow 0$ and $|c(\theta)|$ converges to its maximal value $=\sqrt{r / 2}$ when $\theta \rightarrow 0$ or 1.

Remark 1 (Sign of the wave speed). The sign of the velocity of propagation depends on whether

$\theta<1 / 2$ or $\theta>1 / 2$. In general the sign of $c$ is determined by $F(1)=\int_{0}^{1} f(z) d z$ where $f$ is the reaction term in (1). Indeed the profile $Y$ of a traveling wave is solution of $-Y^{\prime \prime}-c Y^{\prime}=f(Y)$. 
Multiplying it by the derivative of the profile $Y^{\prime}(s)$ (where $Y$ is assumed to satisfy $Y_{-}=0$ and $\left.Y_{+}=1\right)$ and integrating by parts over $\mathbb{R}$, we obtain $-c \int_{-\infty}^{\infty}\left|Y^{\prime}(s)\right|^{2} d s=F(1)$, hence the wave

speed $c$ has an opposite sign with respect to $F(1)=\int_{0}^{1} f(z) d z$, in agreement with the previous observation. This fact allows one to determine the asymptotic value of the solutions in compact sets of $x$ as $t \rightarrow \infty$ : we obtain 0 when $c>0$ and 1 when $c<0$. This observation has been used to develop the asymptotic behaviour of the solutions and the numerical methods in [5].

\subsection{Control to a traveling wave profile}

The birth rate $r$ (positive) of the system is assumed to be known. Without loss of generality, we assume that $r=1$ (up to scaling). We now formulate a control problem for (1). Taking a traveling wave solution as a target, i.e., defining the desired target $y_{d}(x)$ as the value at same time $t=\tau$ of one of the traveling wave solutions above, and given the initial datum $y_{0}$ of the system, our goal is to determine a control law $\theta(t)$ over some time horizon $[0, T]$ (with $T<+\infty$ ) such that the solution $y$ of (1) approaches the target $y_{d}$ at time $T$. Recall that traveling waves are chosen as a target because they describe the dynamics of the interaction particle system (see Section 2) and that they eventually lead to one of the stable steady-states in compact sets as time tends to infinity. The problem can be formulated in various ways:

1. Exact controllability: find $T>0$ and a control strategy $\theta(\cdot)$ over $[0, T]$ such that the corresponding solution satisfies $y(T) \equiv y_{d}$.

2. Approximate controllability: given any $\varepsilon>0$, find $T(\varepsilon)$ (possibly large) and a control strategy $\theta(\cdot)$ over $[0, T(\varepsilon)]$ such that the corresponding solution $y(T(\varepsilon))$ at final time is to an $\varepsilon$ distance of the target $y_{d}$ in $L^{\infty}(\mathbb{R})$ norm. This question can also be addressed by trying to keep the time horizon $T(\varepsilon)$ uniform, independent of $\varepsilon$.

3. Optimal control: a minimisation problem penalising the distance between the state and target.

Exact controllability is an open problem. Here we will establish a result of approximate controllability in large time ensuring that the property above holds for a wide class of initial data. This is anyway at the price that $T(\varepsilon)$ tends to infinity as $\varepsilon \rightarrow 0$. The problem of approximate controllability with a uniform time independent of $\varepsilon$ is also open. Consequently we shall mainly consider the problem within an optimal control perspective.

\subsection{Optimal control problem $(\mathcal{P})_{\text {opt }}$}

We fix a control time horizon $T$ and an open subset $\Omega$ of $\mathbb{R}$. The region $Q=[0, T] \times \Omega$ is the one in which we aim at controlling the system. We consider as a target the traveling wave profile $y_{d}$ corresponding to some of the traveling waves above, i. e., corresponding to some specific choice of the velocity parameter $c$ and $t=\tau\left(y_{d}(x)=Y(x-c \tau)\right)$. For computational purposes the computational domain needs to be finite. We restrict the PDE to $Q$ and we complement it with homogeneous Neumann conditions. We aim to design optimal controls $\theta(t) \in[0,1]$ over $t \in[0, T]$ such that the solution of (1) (with $(t, x) \in Q$ and homogeneous Neumann boundary conditions) starting at a prescribed initial state $y(0, x)=y_{0}(x), y_{0}(x) \in[0,1], x \in \Omega$, is at final time as close as possible to the target state $y_{d}(\cdot)$. The approximate controllability result we will establish shows that this can be done if $T$ is large enough. For practical purposes we are interested in assuring an optimal choice of the control even when $T$ is not large enough. This is why an optimal control strategy is suitable. We consider the optimal control problem $(\mathcal{P})_{\text {opt }}$ consisting of minimising the functional

$$
\mathcal{J}(\theta)=\left\|y(T, \cdot ; \theta)-y_{d}\right\|_{L^{2}(\Omega)}^{2}=\int_{\Omega}\left|y(T, x ; \theta)-y_{d}(x)\right|^{2} d x
$$


over the set $\mathcal{U}_{a d}$ of admissible controls $\theta \in L^{\infty}(0, T)$ satisfying $0 \leqslant \theta(t) \leqslant 1$ for almost every $t \in[0, T]$. The extremal value $\theta=0$ means that there is no Allee effect and $\theta=1$ means that the population decreases for every $y \in[0,1]$. When $\theta=0$ (resp., $\theta=1$ ), the so-called Hair trigger effect occurs (see [3, 4]), meaning that the state $y=0$ (resp., $y=1$ ) is unstable with respect to any positive perturbation, something that may occur under strong human interventions, for instance.

Existence of an optimal control is obvious because of the continuous dependence of solutions of the PDE in $C\left([0, T], L^{2}(\Omega)\right)$ with respect to $L^{\infty}(0, T)$ weak-star convergence of controls. Uniqueness cannot be guaranteed because of the lack of convexity of the functional, due to the nonlinear nature of the PDE under consideration.

Remark 2. As a variant, we could consider the minimisation of $\mathcal{J}=\left\|y-y_{d}\right\|_{Q}^{2}$, aiming to "track" a traveling wave solution $y_{d}(t, x)=Y(x-c t)$ on $Q$ over the time interval [0,T]. However, we formulate here the problem from a controllability point of view, aiming to drive the state to the expected traveling wave at time $T$ without taking care of the values of the state $y(t, x)$ for $t \leqslant T$.

Computational methods. Several methods can be implemented to compute an optimal solution $(\bar{y}, \bar{\theta})$ of $(\mathcal{P})_{\text {opt }}$, including direct or indirect methods. Direct approaches consist of discretizing the optimal control problem to reduce it to a nonlinear programming (NP) problem and then of solving the resulting NP problem by choosing an adequate minimization method (see $[14,36,62,69])$. The advantage of direct methods is that they are easy to implement and they allow to easily include several additional restrictions (such as piecewise constant controls with a given number of pieces, as we shall do here). Direct methods are however computationally expensive and, although they actually suffice to handle 1D problems, gradient-like methods are preferable in multi-D. In the indirect formulation, we start from the fact that the optimal control $\bar{\theta}$ is characterised by the following variational inequality (in weak formulation, see [52, 70])

$$
\mathcal{J}^{\prime}(\bar{\theta}) \cdot(v-\bar{\theta})=\iint_{Q} \bar{p}(t, x) \partial_{\theta} f(\bar{y}, \bar{\theta}) \cdot(v-\bar{\theta}) d x d t \geqslant 0, \quad v \in \mathcal{U}_{a d}
$$

and the gradient of the cost functional can be computed with the adjoint system

$$
\begin{aligned}
\partial_{t} p(t, x)+\partial_{x x} p+p \partial_{y} f(\bar{y}, \bar{\theta})=0, & (t, x) \in Q, \\
\partial_{x} p(\cdot, x)=0, & x \in \partial \Omega, \\
p(T, x)=2\left(\bar{y}(T, x)-y_{d}\right), & x \in \Omega .
\end{aligned}
$$

The optimality system consists of the state equation (1), of the adjoint equation (9) and of the optimality condition (8). It can alternatively be obtained by applying the Pontryagin Maximum Principle (see [61]). To solve numerically the optimality system explicitly one may use iterative descent (gradient-type) methods (see [17]). The descent direction $d^{k}$ at step $k$ is chosen such that $\mathcal{J}^{\prime}\left(\theta^{k}\right) \cdot d^{k} \leqslant 0$ and $\theta^{k} \in \mathcal{U}_{a d}$.

\subsection{Extended optimal control problem $(\mathcal{P})_{S I R M}$}

As mentioned in Section 2.3, it is interesting to consider the optimal control problem for (3) in which the Allee threshold is controlled indirectly by a second equation. We consider $u$ to be the control of the system, the value of $\theta$ and $r$ being fixed. Even if $\theta$ is fixed in (2.3), the effective value of the Allee threshold for the total population $y=y_{1}+y_{2}$ in (4) is $\theta+y_{2}$, so that the action of the control $u$ on (3) affects the Allee threshold similarly as the control $\theta$ for the scalar system considered in the previous section does. According to the stochastic model (5), $u$ is the "birth" rate of the sterile couple: at rate $u$, a normal couple transforms to a sterile one. Moreover, it is natural to assume that $u=u(t)$ is time-dependent because it can be tuned by human interactions (such as changing the release rate of sterile males). 
Fix a final time $T$ and a bounded open subset $\Omega$ of $\mathbb{R}^{2}$. Let $Q=\Omega \times[0, T]$. We consider the following optimal problem $(\mathcal{P})_{S I R M}$ consisting of minimizing the functional

$$
\mathcal{J}(u)=\frac{1}{2} \iint_{Q} y(t, \mathbf{x})^{2} d \mathbf{x} d t+\frac{1}{2} K \int_{0}^{T}|u(t)|^{2} d t
$$

where $K>0$ is a positive number and $\mathbf{y}$ stands for the solution of (3) satisfying a given the initial condition $\mathbf{y}(0, \mathbf{x})=\mathbf{y}_{0}=\left(y_{10}, y_{20}\right)^{\top}, \mathbf{x} \in \Omega$, and homogeneous Neumann-boundary conditions, over the class $\mathcal{U}_{a d}$ of all possible controls satisfying $0 \leqslant u(t) \leqslant u_{\max }$ for almost every $t \in[0, T]$, for some $u_{\max }>0$. Recall that $y=y_{1}+y_{2}$ represents the total density of the population. The cost functional (10) is motivated by the fact that we aim at using the sterile male release method to eradicate the pest population. By doing so, we search for attaining the objective by releasing as less sterile males as possible (since it is very expensive to rise numerous sterile males). Both terms in the functional aim at minimising the total population density and the total number of sterile males released.

The existence of an optimal control for this problem is as well obvious but uniqueness may fail because of the lack of convexity of the functional (see [7,63]). The optimal control $\bar{u}$ must satisfy (see $[21,63,70]$ )

$$
\bar{u}(t)=\min \left(\max \left(0,-\frac{1}{K} \int_{\Omega} \mathbf{p}^{\top} \frac{\partial F(\mathbf{y}, u)}{\partial u} d \mathbf{x}\right), u_{\max }\right)
$$

where $\mathbf{p}=\left(p_{1}, p_{2}\right)^{\top}$ is the adjoint state which solves the adjoint system

$$
\frac{\partial}{\partial t} \mathbf{p}+\triangle \mathbf{p}+\left(\frac{\partial F}{\partial \mathbf{y}}\right)^{\top} \mathbf{p}+\mathbf{y}=0
$$

with homogeneous Neumann-boundary conditions and terminal condition $\mathbf{p}(T, \mathbf{x})=(0,0)^{\top}$ for every $\mathbf{x} \in \partial \Omega$. These key facts play a role when implementing descent algorithms for the numerical approximation of the minimisers of $(\mathcal{P})_{S I R M}$, requiring in particular to solve (1) forward and (12) backward in time but keeping (11). Several acceleration methods can also be implemented, combining numerical grids, first solving the problem with a less precise time-space discretization, to later do it in a finer time-space mesh while using the obtained less precise solution. The term $\frac{1}{2} K \int_{0}^{T}|u(t)|^{2} d t$ in (10) of the cost functional in $(\mathcal{P})_{S I R M}$ can be viewed as a Tikhonov regularisation term (see [60]), enhancing (but not guaranteeing) convexity of the cost functional and, in view of the possible non-uniqueness of the critical points, it may help at varying the local minimum achieved by means of the descent algorithm (see [29]).

\section{Approximate controllability in large time}

This section is devoted to establish approximate controllability properties in large time, exploiting the well known asymptotic stability properties of the reaction-diffusion system under consideration. We first do it when the target is a traveling wave to later consider the case where the target is the superposition of two traveling fronts propagation in opposite directions.

\subsection{Control to a traveling wave target}

Let $r>0$ be arbitrarily fixed.

Proposition 1. Let the target $y_{d}$ of the control problem be the profile $Y$ of the traveling wave solution (6) with center at $s_{0}=x_{d}$. Assume that the initial data $y_{0}$ satisfies

$$
\lim _{x \rightarrow-\infty} \sup y_{0}(x)<\lim _{x \rightarrow+\infty} \inf y_{0}(x) .
$$


Then, for every $\varepsilon>0$ there exist $T(\varepsilon)>0$ (large enough) and infinitely many piecewise constant control strategies steering the system (1) from $y_{0}$ to a $\varepsilon$-neighborhood (in $L^{\infty}(\mathbb{R})$ norm) of $y_{d}$ in time $T(\varepsilon)$ (approximate controllability in large time). Moreover, if $y_{0}$ is a translation of the traveling wave profile (6) then the target $y_{d}$ can be reached exactly (exact controllability in large time).

Proof. We proceed in several steps.

Step 1. Asymptotic behaviour with constant control $u(t) \equiv \theta$.

The following uniform convergence result, which is a consequence of [32, Theorem 3.1] (which applies to a wider class of nonlinearities than the cubic bistable one considered here) will be useful.

Lemma 1. ([32]) If the initial datum $y_{0}$ satisfies $y_{0}(x) \in[0,1]$ for every $x \in \mathbb{R}$ and satisfies the condition (13) and thus

$$
\liminf _{x \rightarrow+\infty} y_{0}(x)>\theta, \quad \limsup _{x \rightarrow-\infty} y_{0}(x)<\theta
$$

for some $0<\theta<1$, then, for some $x_{1} \in \mathbb{R}$, the solution of

$$
\begin{aligned}
& y_{t}-y_{x x}=r y(1-y)(\theta-y), \quad x \in \mathbb{R}, \quad t>0, \\
& y(0, \cdot)=y_{0}
\end{aligned}
$$

approaches a unique traveling wave $Y\left(x-c t-x_{1}\right)$ uniformly in $x$ and exponentially in time for $c=c(\theta)$ as in (7), i.e., for some positive constants $K$ and $\gamma$, the solution $y$ of (1) satisfies

$$
\left\|y(t, x)-Y\left(x-c t-x_{1}\right)\right\|_{L^{\infty}(\mathbb{R})}<K e^{-\gamma t} .
$$

This result shows that by taking the constant control $u(t) \equiv \theta$, with $\theta$ satisfying (14), the state converges as $t \rightarrow \infty$ to a translation of the traveling wave profile and thus is close to it within a large enough horizon of time: whatever $\varepsilon>0$ is, for $T(\varepsilon)$ large enough, the solution is at $\varepsilon$-distance of a translation of the traveling wave profile. Note however that the actual location $c T(\varepsilon)+x_{1}$ of the center of the target profile and, in particular, the translation factor $x_{1}$ depends on the nature of the initial datum. Observe also that the time needed in this first step $T(\varepsilon)$ depends logarithmically on $\varepsilon$ :

$$
T(\varepsilon) \sim \log (\varepsilon / K) .
$$

Step 2. Adjustment of the center of the traveling wave profile.

Once at time $t=T(\varepsilon)$ at which the solution $y$ is at $\varepsilon$-distance of the traveling wave profile translated to $s=c T(\varepsilon)+x_{1}$, noting that the target is the same profile but translated to $s=x_{d}$, and in view of the fact that the profiles of the traveling waves are all the same for all values of $\theta$ and that only their velocity of propagation changes as a function of $\theta$, it is natural to control the system by "pushing" the profile from the center $s=c T(\varepsilon)+x_{1}$ to $s=x_{d}$. Of course, in the unlikely case where $c T(\varepsilon)+x_{1}=x_{d}$ this second step is not necessary. But otherwise, by taking a constant control $u(t) \equiv \theta^{\prime}$ with a convenient choice of the sign of $\theta^{\prime}-1 / 2$, depending on whether $x_{d}$ is to the left or to the right of $c T(\varepsilon)+x_{1}$, in view of the velocity of propagation formula (7), in the time interval $t \in\left[T(\varepsilon), T(\varepsilon)+\tau\left(\theta^{\prime}\right)\right]$ with $\tau\left(\theta^{\prime}\right)=\left|c T(\varepsilon)+x_{1}-x_{d}\right| / c\left(\theta^{\prime}\right)$, the traveling wave with center at $s=c T(\varepsilon)+x_{1}$ at time $T(\varepsilon)$ and with control $u \equiv \theta^{\prime}$ moves to the one with center in $s=x_{d}$ in time $T(\varepsilon)+\tau\left(\theta^{\prime}\right)$.

This very simple argument shows that the traveling wave that at time $T(\varepsilon)$ was close to the actual solution and gets exactly to the target traveling wave profile. But we need however to analyze what is the evolution of the actual solution of the state departing from $y(T(\varepsilon), x)$ in time $t=T(\varepsilon)$ and with control $u \equiv \theta^{\prime}$ in time $t \in\left[T(\varepsilon), T(\varepsilon)+\tau\left(\theta^{\prime}\right)\right]$.

At this point it is convenient to observe how the velocity of propagation of the traveling wave depends on the value of the parameter $\theta$. When $\theta \rightarrow 1 / 2$ the velocity tends to zero. At the 
contrary, when $\theta$ tends to the extremal values 0 or 1 , the velocity is maximised wit absolute value $c=\sqrt{r} / 2$. It is then convenient choose $\theta^{\prime}$ near these extremal velocities so that the length of the second time interval is kept as small as possible. Note however that, in view of (16), the length of this second time interval is of the order of $\tau\left(\theta^{\prime}\right) \sim \log (\varepsilon / K) / \gamma$ as well.

The control in this second step has been chosen to ensure the exact transport of the center of gravity of the traveling wave. Let us now explain how it affects the actual solution. Using a Gronwall type estimate and taking into account that the nonlinearity of the system with $u \equiv \theta^{\prime}$ is globally Lipschitz, it is not difficult to see that the distance between the actual state and the traveling wave profile at time $t=T(\varepsilon)$, which is $\sim \varepsilon$, is amplified by a factor of the order of $C_{1} \exp \left(C_{2} \tau\left(\theta^{\prime}\right)\right) \sim C_{1} \exp \left(C_{2} \log (\varepsilon / K) / \gamma\right) \sim C^{\prime}$ independent of $\varepsilon$. In other words we have

$$
\left\|y\left(T(\varepsilon)+\tau\left(\theta^{\prime}\right)\right)-y_{d}\right\|_{L^{\infty}(\mathbb{R})} \leqslant C^{\prime} \varepsilon .
$$

Step 3. Conclusion.

This argument applied with $\varepsilon^{\prime}=\varepsilon / C^{\prime}$ completes the proof, ensuring that the final distance (17) is less than $\varepsilon$. Of course when the initial datum $y_{0}$ coincides with a translation of the traveling wave profile the first step of the proof is not needed and the second one ensures that the solution reaches exactly the target in finite time.

Remark 3. We have established approximate controllability in large time with piecewise constant controls consisting of two pieces. As shown above the choice of controls of this form is not unique since the values of $\theta$ and $\theta^{\prime}$ are not uniquely determined. But in the second step we could choose a more complex control made of several constant pieces, for different values of $\theta^{\prime}$, the essential criterion being to move the center of the traveling wave profile from $c T(\varepsilon)+x_{1}$ to $x_{d}$.

\subsection{Control to a pair of opposing traveling fronts}

Control objectives can be addressed for more general targets $y_{d}$ that are for instance the superposition of a pair of opposing traveling waves:

$$
y_{d}(x)=\frac{e^{\sqrt{r / 2}\left(x-x_{d}^{-}\right)}}{1+e^{\sqrt{r / 2}\left(x-x_{d}^{-}\right)}}+\frac{e^{\sqrt{r / 2}\left(-x-x_{d}^{+}\right)}}{1+e^{\sqrt{r / 2}\left(-x-x_{d}^{+}\right)}}-1
$$

with prescribed values of $x_{d}^{-}$and $x_{d}^{+}$. In this case the target function is not the trace at some given time $t$ of a solution since the superposition of two traveling waves is not solution of (1). The following result is a consequence of [32, Theorem 3.2].

Lemma 2. ([32]) Assume that $\theta<1 / 2$ and that the initial datum $y_{0}$ satisfies $0 \leqslant y_{0}(x) \leqslant 1$ for every $x \in \mathbb{R}$ and

$$
\liminf _{|x| \rightarrow+\infty} y_{0}(x)<\theta, \quad y_{0}(x)>\theta+\eta \text { for }|x|<M
$$

for some positive real numbers $\eta$ and $M$. If $M$ (which depends on $\eta$ and $\theta$ ) is large enough then there exist $x^{-} \in \mathbb{R}, x^{+} \in \mathbb{R}, K>0$ and $\beta>0$ such that the solution of (15) develops (uniformly in $x)$ into a pair of diverging traveling fronts $Y\left(x-c(\theta) t-x^{-}\right)+Y\left(-x-c(\theta) t-x^{+}\right)-1$, i.e.,

$$
\begin{aligned}
& \left|y(t, x)-Y\left(x-c(\theta) t-x^{-}\right)\right|<K e^{-\beta t}, \quad x<0, \\
& \left|y(t, x)-Y\left(-x-c(\theta) t-x^{+}\right)\right|<K e^{-\beta t}, \quad x>0 .
\end{aligned}
$$

This result means that the $x$-interval on which the solution is close to 1 is elongating in both directions with speed $c(\theta)$. This guarantees in particular that when $y_{0}$ is the characteristic function of a large enough interval the solution develops the structure of two propagating fronts so that eventually the solution tends to the steady-state $y=1$ as $t \rightarrow+\infty$. When $\theta>1 / 2$ a similar 
phenomenon occurs by exchanging the roles of the two stable equilibria 0 and 1 . When $y_{0}$ is even with respect to some $x_{0}$ the solution $y(t, \cdot)$ is even as well with respect to $x_{0}$ for every $t>0$.

It is anyway not as simple as in the previous section to regulate the actual location of the two fronts by means of piecewise constant controls to achieve approximate controllability in large time. This problem is open from an analytical viewpoint. For this reason the use of computational methods is particularly suitable in this context.

\section{$5 \quad$ Numerical simulations}

In this section we solve numerically the optimal control problems introduced previously. We have seen that traveling wave solutions play an important role in the dynamics of the models and that, in some particular cases, piecewise constant controls may suffice to control the system arbitrarily close to such profiles in large time. We are going to perform numerical simulations for given time horizons that are not necessarily large.

\subsection{The one-dimensional case}

Recall that direct methods of optimal control consist of first discretising the problem to reduce it to a finite-dimensional nonlinear programming (NP) problem, to be then solved by some numerical optimisation technique. We discretise the dynamics with spatial finite differences and a second-order implicit Crank-Nicolson scheme in time, which is unconditionally stable (see [66]). The control could be discretised according to the same time-grid but this would increase in an unnecessary way the computational cost in the implementation of the optimisation method, taking into account that, in practice, we seek controls that are piecewise constant with only a few pieces.

There are many available methods for solving NP problems. We use here the modeling language and automatic differentiation software AMPL (see [37]) combined with the interior point optimisation routine Ip0pt (see [69, 71]).

Our objective is to minimize the functional

$$
\mathcal{J}(\bar{\theta})=\left\|y(T, \cdot)-y_{d}\right\|_{L^{2}(\Omega)}^{2}
$$

with $\Omega=(-L, L)$ for some $L>0$. Numerical results are provided in two different cases. In Case A we investigate problems where the initial data $y_{0}$ is a characteristic function and the expected target $y_{d}$ is a pair of diverging traveling waves and the time-horizon is large enough. In Case B we analyse the same problem when the time horizon $T$ is not too large, in which case the analytical results of the previous section, based on the asymptotic stability properties of the system may not apply. Note that the time being short or large depends actually on the nature the initial datum as compared to that of the target since, as our analysis above shows, the effect of the control parameter $\theta$ allows only to enhance propagation properties up to an upper limit.

Case A: large time. We set $T=30$. Assume that the expected wave $y_{d}$ is the pair of traveling waves given by (18) with $r=1, x_{d}^{-}=-x_{d}^{+}=-14.2426$ and $L=30$. We take

$$
y_{0}(x)=\left\{\begin{array}{l}
\theta_{0}-0.01, \quad x \in[-5,5] \\
0, \quad \text { otherwise }
\end{array}\right.
$$

with $\theta_{0}=0.7$ (see the shape of $y_{0}$ on Fig. 4 , in the middle, with black dotted solid line).

Applying our numerical method with $N=1$ (so that the control is a constant function) and initialising the problem with the control 0.275 , we obtain the constant control

$$
\bar{\theta} \equiv 0.2513,
$$

with the corresponding cost $\mathcal{J}(\bar{\theta})=2.3587 \times 10^{-4}$. 
When $N=2$, i.e., allowing the control to be made of two constant pieces, we obtain

$$
\bar{\theta}(t)=0.2177, \text { for } t \in\left[0, t_{1}\right), \quad \bar{\theta}(t)=0.2813, \text { for } t \in\left[t_{1}, T\right],
$$

with $t_{1}=14.6999$ and the corresponding cost $\mathcal{J}(\bar{\theta})=2.3573 \times 10^{-4}$. The solutions corresponding to $N=2$ of the controlled and the uncontrolled system can be observed on Fig. 4. The sub-figure in the middle shows that the final state $y(T, x)$ is very close to the expected wave $y_{d}$ in $\Omega$. The state $y$ develops first to $y\left(t_{1}, x\right), x \in \Omega$ (green line), which takes the form of a pair of diverging traveling waves, to later move to the expected $y_{d}$. As the simulation of the solution of the problem with $\theta=\theta_{0}$ shows (see the right sub-figure of Fig. 4), extinction occurs if the system is not controlled due to the threshold effect. The computed control, accordingly, allows avoiding efficiently the extinction phenomenon and to control the system towards the chosen target. Even if the time horizon is not large enough to apply the approximate controllability results in large time of the previous section, the computational results show the feasibility of the piecewise constant strategy.
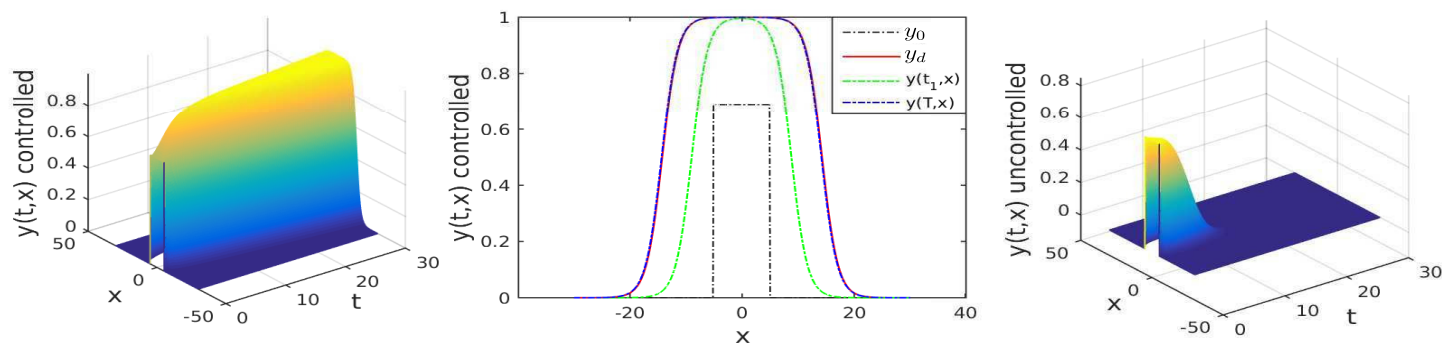

Figure 4: Left and middle: state evolution of the controlled $(N=2)$ system. Right: state evolution of the uncontrolled system.

Case B: short time. We set again $T=30$ but we adjust $x_{0}, x_{d}^{+}$, and $x_{d}^{-}$so that the expected wave $y_{d}$ is far enough from the initial data $y_{0}$. We observe two different situations.

The first situation is shown on Fig. 5. Here we have set $x_{d}^{-}=-x_{d}^{+}=-24.2426$, which makes $y_{d}$ (red solid line) "far" from the initial data $y_{0}$ (black dashed line). The problem is solved on $\Omega=(-50,50)$. The optimal controls obtained by setting $N=1$ and $N=2$ are the same $: \bar{\theta} \equiv 0$. The corresponding cost is $\mathcal{J}(\bar{\theta})=0.1369$.

Recall that when $\theta=0$ (or $\theta=1$ ), the wave has its maximal speed according to (7). This means that the optimal control strategy consists of pushing the system solution towards the expected wave $y_{d}$ with a maximal speed.

The second situation is shown on Fig. 6. Here we have changed the initial data to be $y(x)=$ $\theta_{0}-0.01$ for $|x| \leqslant 40$ and $y(x)=0$ for the rest. Applying the numerical method with $N=2$, we obtain

$$
\bar{\theta}(t)=0.6925, \text { for } t \in\left[0, t_{1}\right), \quad \bar{\theta}(t)=0.6171, \text { for } t \in\left[t_{1}, T\right],
$$

with $t_{1}=15$ and the corresponding $\operatorname{cost} \mathcal{J}(\bar{\theta})=0.1386$. In contrast to the solution on Fig. 5 , the final state $y(T, x)$ on Fig. 6 is not a translation of the expected wave $y_{d}$.

We see on Fig. 5 that the state converges first to a translation of the expected wave and then moves as rapidly as possible towards the expected wave. This is consistent with our intuition and 

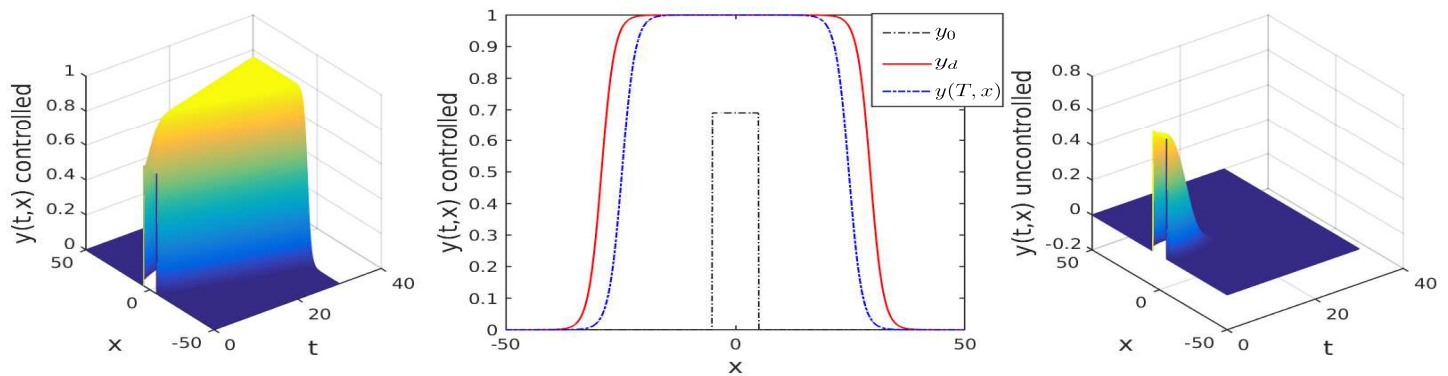

Figure 5: Left and middle: state evolution of the controlled system. Right: state evolution of the uncontrolled system.
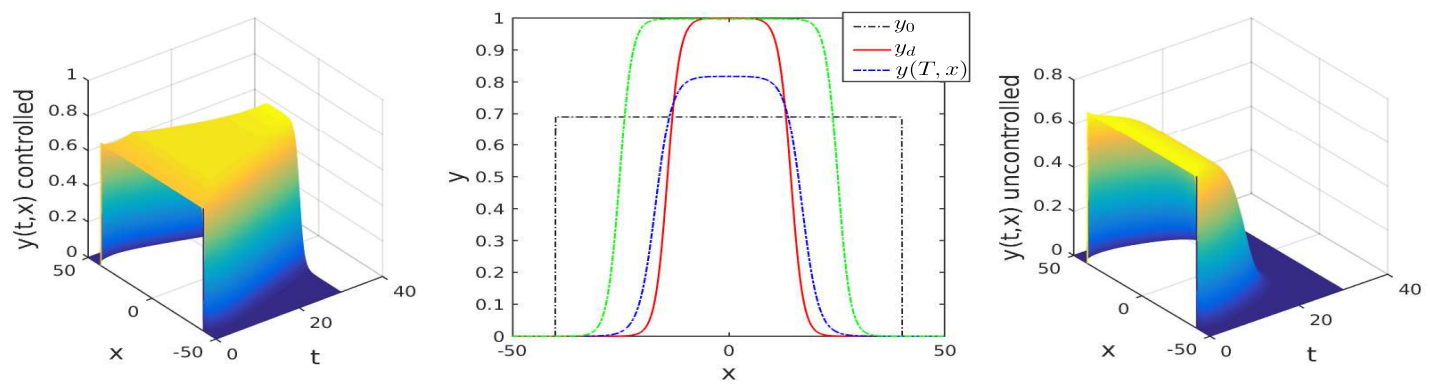

Figure 6: Left and middle: state evolution of the controlled system (the green line in the middle sub-figure represents a final state $y(T, x)$ associated to a non-optimal control $\theta$ ). Right: state $y$ of uncontrolled system.

with the controls that we have constructed (when the final time $T$ is large enough): first "wait" that the state has developed into a translation of the traveling wave and then "push" it towards the expected wave. However, the solution we observe on Fig. 6 is rather against this intuition: it has made a compromise between "shape" and "position". Actually if we apply our intuitive control strategy to the system (letting $\theta(t)=0$ for $t \in[0,5]$ and $\theta(t)=1$ for $t \in\left(5, t_{f}\right]$ ), we get the final state $y(T, x)$ (plotted by the green dashed line in the middle sub-figure of Fig. 6 ) which is quite far from $y_{d}$ (plotted by red line). The corresponding cost is $\mathcal{J}(\theta)=1.6140$, which shows clearly that this strategy is not better than the one derived by the algorithm.

Note that it is the propagation direction that makes these two situations different. In fact, the value $\theta=0$, which helps the state $y$ to converge to a translation of the expected wave, makes the state 1 to invade the space. In the first situation (Fig. 5), the state $y$ develops into a pair of diverging traveling waves quickly and then continues to propagate outwardly with $\theta=0$. However, in the second situation (Fig. 6), the state has to change the propagation direction after converging to a pair of diverging traveling waves, because to get closer to $y_{d}$, it is the state 0 instead of the state 1 that should propagate. Therefore, the intuitive control strategy actually makes the state $y$ to firstly get further to $y_{d}$, and hence it may be not better than a control strategy that makes a compromise between loss of shape and loss of position. 


\section{$5.2 \quad$ Two-dimensional case}

Now let us solve numerically the extension problem $(\mathcal{P})_{S I R M}$ formulated in Section 3.4. We do it using a gradient algorithms who is more suitable in $2 \mathrm{D}$ when dealing with more complicated dynamics and patterns. We show by a numerical example that the Allee threshold $\theta$ can be controlled concretely in practice and that the Allee effect can indeed be used to reach our control objective.

Uncontrolled system solution. We set $\Omega=[-15,15]^{2} \subset \mathbb{R}^{2}$. We take the birth rate $r=1$ and the Allee effect $\theta=0.1$, and

$$
\mathbf{y}_{0}(\mathbf{x})=(1,0)^{\top}, \text { if }\left|x_{i}\right| \leqslant 2, i=1,2
$$

and $\mathbf{y}_{0}(\mathbf{x})=(0,0)^{\top}$ for other points $\mathbf{x}$ in $\Omega$. Then, by numerically integrating the uncontrolled system, we obtain the solution shown on Fig 7 . Without control, i.e., $u \equiv 0$, the density $y_{1}$ converges to 1 in the whole domain by means of a radial wave solution. It is therefore natural to analyse the control problem aiming to eliminate the pest population.
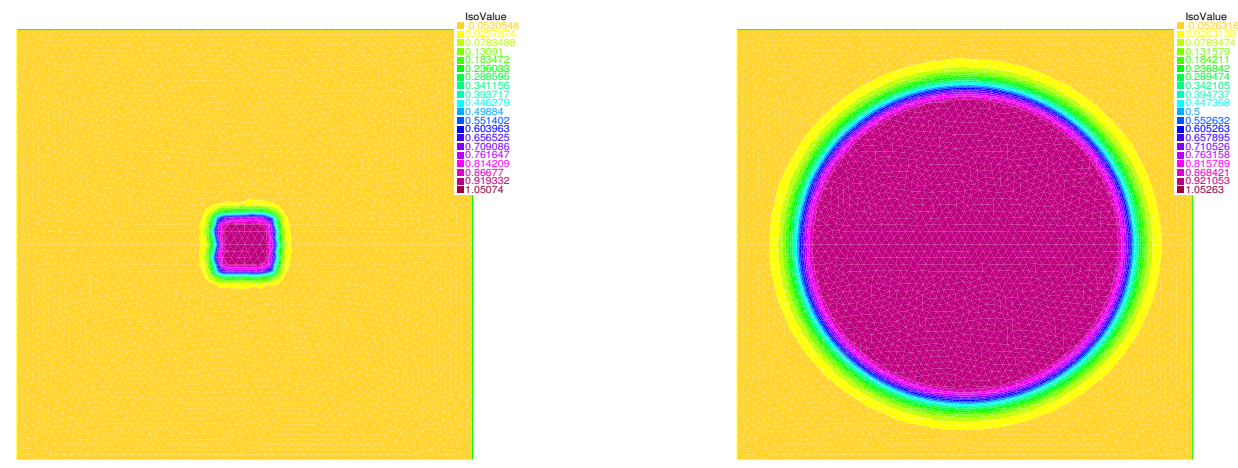

Figure 7: State $y_{1}(t, \mathbf{x})$ with $u \equiv 0$ at time 0 (left) and at time 60 (right).

Optimal control solution. We take $T=70, K=10$ and $u_{\max }=0.2$. Initializing the numerical method with $u \equiv 0.15$, we obtain the optimal solution control shown on Fig. 8 and the associated final state $\mathbf{y}(T, x)$ on Fig. 9. Here we have used the BFGS method (see [17]) to approximate the inverse of the Hessian matrix and the software Freefem++ (see [39]) to solve (3) and (12).

Control with Allee Effect. In the above example, at time $t=42$, the maximal value of $y_{1}=0.097$ is already smaller than $\theta=0.1$. According to the Allee threshold effect, we know that $y_{1}$ will tend to zero even without $y_{2}$. This means that for $t>42$ the sterile population is no longer needed to reach our objective of getting rid of the pest population. In fact, at some point, the Allee effect becomes strong enough to perform alone the elimination mission. For example when applying the truncated optimal control (see right sub-figure on Fig. 8) $u(t)=u^{*}(t)$ for $t \leqslant 25$ and $u(t)=0$ for $t>25$ the solution $\mathbf{y}$ at the same final time $T=70$ (see Fig. 10) is still nearly zero. Comparing the cost, when using the optimal control $\bar{u}$ shown on Fig. 8, the two terms in the cost functional (10) are $\frac{1}{2} \iint_{Q} y^{2} d x d t=3.78$ and $\frac{1}{2} \int_{0}^{T}|\bar{u}(t)|^{2} d t=3.56$. When using the truncated control $u$ shown on Fig. 10, we have $\frac{1}{2} \iint_{Q} y^{2} d x d t=3.94$ and $\frac{1}{2} \int_{0}^{T}|\tilde{u}(t)|^{2} d t=3.53$. The cost 

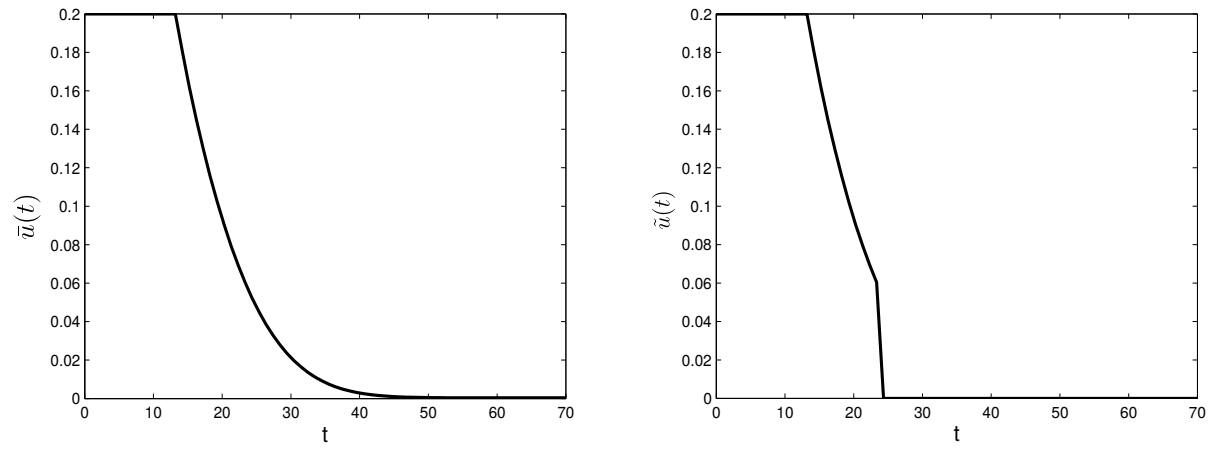

Figure 8: Left: optimal control $\bar{u}(t)$. Right: truncated optimal control $\tilde{u}(t)$.
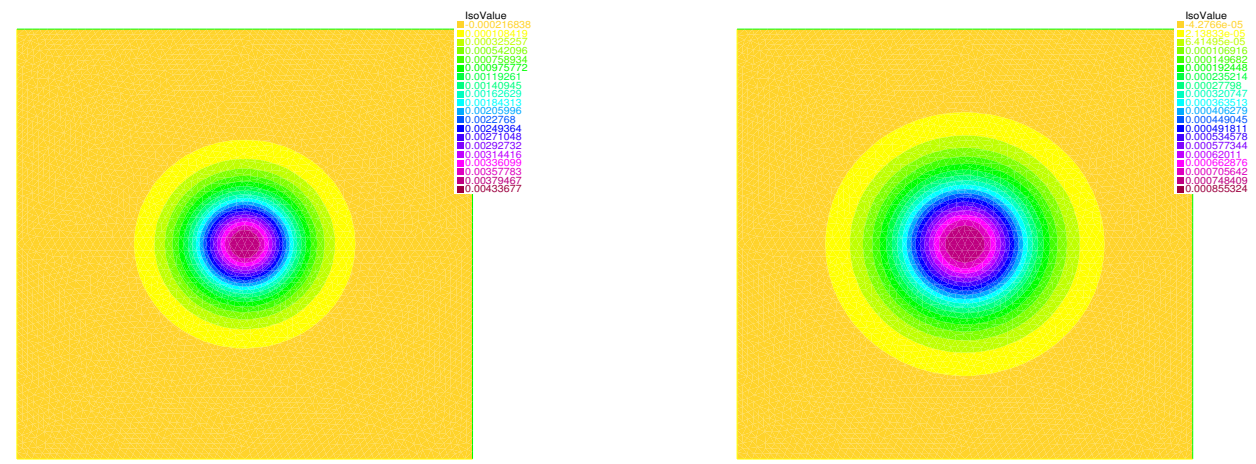

Figure 9: Optimal final state $y_{1}(T, x)$ (left) and $y_{2}(T, x)$ (right).

(10) with $\bar{u}$ is smaller but more sterile pests are required. More precisely, when applying $\bar{u}$ we have $\iint_{Q} y_{2}(t, x) d x d t=19.9$ while $\iint_{Q} y_{2}(t, x) d x d t=17.35$ when using $\tilde{u}$.

\section{Deterministic control of the stochastic systems}

In this section we numerically investigate how the stochastic systems (2) and (5) behave when applying the optimal controls obtained for the macroscopic PDE models (1) and (3) (which are the mean field limits of the microscopic models). Numerical simulations show that the optimal controls of $(\mathcal{P})_{\text {opt }}$ and of $(\mathcal{P})_{S I R M}$, when applied to the corresponding interactive particle systems, give satisfactory results. It would be interesting to analyse this question from a theoretical viewpoint too.

We first apply the optimal control obtained for Case A in Section 5 to the interacting particle system (2). We take $\mathbb{Z}=[-600,600]$ and $\varepsilon=0.05$. The "mean" population distributions of 100 times of simulations (see Section 2.2) are shown on Fig. 11. The green and blue lines represent the initial data (19) and the expected wave pair $y_{d}$ shown on Fig. 4 and the black and red lines represent the simulation results of the stochastic model (2).

We next apply the optimal control obtained in Section 5.2 to the stochastic model (5). If $\theta \equiv 0$ and $y_{2}(0, \cdot)=0$ (meaning that no sterile males are added to the system) then the equation for $y_{1}$ is the same than (1) in dimension two. In the example of Fig. 2, the pest population $y_{1}$ will increase with time and thus controls must be used to eliminate the pest population. Applying the optimal control $\theta^{*}(t)$ shown on Fig. 8 to the system (5), we see on Fig. 12 the evolution of the number of 

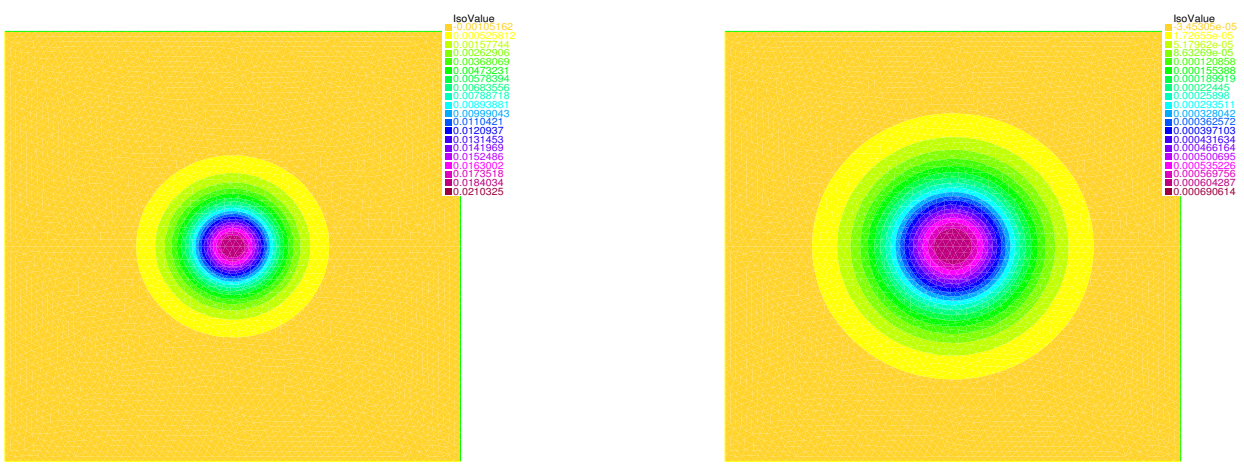

Figure 10: Final state $y_{1}(T, x)$ (left) and $y_{2}(T, x)$ (right) with truncated optimal control.
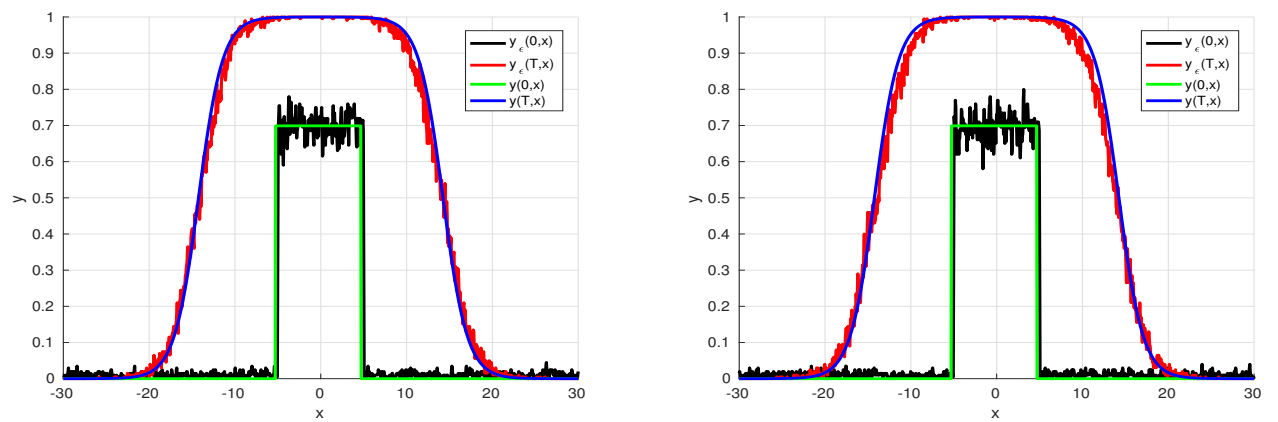

Figure 11: Comparison of solutions obtained from macroscopic and stochastic controlled models. Left: obtained by applying control (20). Right: obtained by applying control (21)

normal and sterile populations and the target population is eliminated with this control.

Recall that a solution of the deterministic model represents a "mean" behaviour of the stochastic model. Hence for having a better matching' with a radial wave solution of (3) we need to simulate a large number of times (5) and calculate an expectation of these solutions.

Conversely the question would be interesting to determine optimal controls for the microscopic models and to see if they withstand a passage to the limit, tending to controls for the macroscopic models (see $[2,35,45]$ ).

\section{Conclusion, open problems and perspectives}

In this paper we have considered an optimal control problem for a $1 \mathrm{D}$ reaction-diffusion model arising in ecology. Inspired by control methods implemented in ecology practice such as sterile male and mating disruption techniques, taking advantage of the threshold effect, we have shown that the Allee threshold $\theta$ can be used as a control to act on the population distribution. The models we considered and results we obtained are also relevant in other applications, such as mathematical biology, chemistry, social sciences, etc. The PDE macroscopic model we considered has been derived as a mean field limit of a stochastic model for individual behaviour. The suitability of this link has been illustrated by numerical simulations. A richer system of reaction-diffusion equations has also been introduced to show that actuating through the threshold parameter is analytically realistic. We have constructed piecewise constant controls driving solutions to traveling wave profiles in large time, using known asymptotic and stability properties of traveling waves. A direct computational 


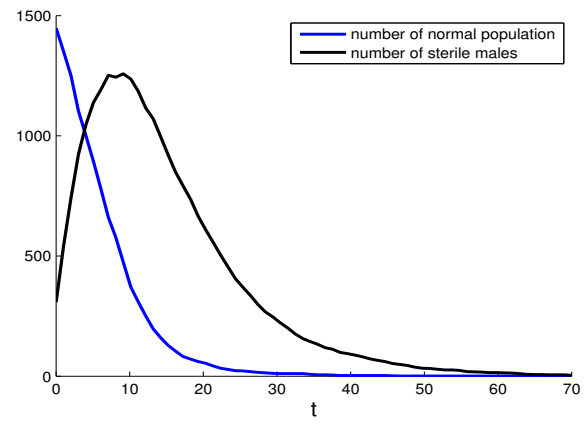

Figure 12: Evolution of the number of normal and sterile populations.

method has been developed to numerically compute such optimal controls. This method provides also satisfactory solutions for shorter time intervals for which the control theoretical results based on the asymptotic properties of solutions do not apply. The methods developed in this paper can also be applied in the multi-dimensional case where the dynamics exhibit added geometric complexity.

We end the paper by listing hereafter a number of open problems arising in this context.

Controllability issues. We have established approximate controllability in large time to a traveling wave profile. Can approximate controllability be proved within a time that is independent of the distance $\varepsilon$ ? Can the target be reached exactly? In contrast to classical controllability results for parabolic equations by means of additive controls, in the present context controllability is not expected to occur within arbitrarily small time since, roughly, the choice of the $\theta$ parameter only affects the velocity of propagation of traveling fronts, which is uniformly limited. In this context the methods developed in [19] could be useful. Approximate controllability in large time could be investigated when the target is a pair of opposing traveling fronts.

Sparse controls. In this paper the control has been applied through the threshold parameter. By means of analytical and computational results we have shown that a piecewise constant strategy can control the system, in particular to a prescribed traveling wave target. Control of traveling waves has been investigated in [7] in multi-D by means of additive controls and optimal control strategies are developed with the aim of building sparse controls with a narrow support. The results of [7] show that optimal controls have a localised moving support near the locations where the traveling fronts reach maximal values. The links between these results and ours, relying on the use of the Allee threshold effect, would be worth to be explored.

General patterns. The control problems addressed in this paper also make sense for more general multi-D models in which target solutions have a richer geometry such as radial waves or Turing patterns. General kinetic models arise also naturally in this context. Their analysis from a control perspective in relation with population dynamics issues is an open subject. Controllability issues are interesting and problems can be formulated within an optimal control viewpoint.

Stochastic models. The control of stochastic models and their limiting behavior when taking mean field limits towards the controls of the diffusive models considered in this paper could also be investigated more rigorously, following the modelling approach presented in Section 2. 
Acknowledgment. The third author thanks J.R. Uriarte from the Faculty of Economics of the University of Basque Country (UPV/EHU) who raised the problem of modelling and control in the context of the of multilinguism. His advice played a key role to define the work program that led to the research we describe in this paper.

Authors thank also Etienne Adam for his help in the stochastic interpretation of the microscopic models.

This research was conducted while the second author was a postdoctoral fellow of the Laboratoire Jacques-Louis Lions, funded by the ICON project of the French ANR-16-ACHN-0014. This work was also partially funded by the Advanced Grant DyCon (Dynamical Control) of the European Research Council Executive Agency (ERC) and the MTM2014-52347 and MTM2017-92996 Grants of the MINECO (Spain).

\section{References}

[1] G. Albi, L. Pareschi, G. Toscani, M. Zanella, Recent advances in opinion modeling: control and social influence. In Active Particles, Springer International Publishing, 2017, vol. 1, p. 49-98.

[2] M. Annunziato, A. Borzì, A Fokker-Planck control framework for multidimensional stochastic processes. Journal of Computational and Applied Mathematics, 2013, vol. 237, no 1, p. 487-507.

[3] D.G. Aronson, H.F. Weinberger, Nonlinear diffusion in population genetics, combustion, and nerve pulse propagation, in Partial Differential Equations and Related Topics, Lecture Notes in Math., Springer, Berlin, 1975, vol. 446, p. 5-49.

[4] D.G. Aronson, H.F. Weinberger, Multidimensional nonlinear diffusions arising in population genetics, Adv. Math., 1978, vol. 30, p. 33-76.

[5] J.M. Arrieta, M. Lopez-Fernandez, E. Zuazua, Approximating travelling waves by equilibria of nonlocal equations, Asymptotic Analysis, 2012, vol. 3, no. 78, p. 145-186.

[6] A. Aydogdu, M. Caponigro, S. McQuade, B. Piccoli, N.P. Duteil, F. Rossi, E. Trélat, Interaction Network, State Space, and Control in Social Dynamics. In Active Particles, Springer International Publishing, 2017, vol. 1, p. 99-140.

[7] W. Barthel, C. John, F. Tröltzsch, Optimal boundary control of a system of reaction diffusion equations. ZAMM-Journal of Applied Mathematics and Mechanics, 2010, vol. 12, no. 90, p. 966-982.

[8] N. Barton, M. Turelli, Spatial waves of advance with bistable dynamics: cytoplasmic and genetic analogues of Allee effects, The American Naturalist, vol. 178, no. 3, pp. E48-E75, 2011.

[9] K. Beauchard, M. Morancey, Local controllability of 1D Schrödinger equations with bilinear control and minimal time, arXiv preprint arXiv:1208.5393, 2012.

[10] N. Bellomo, L. Preziosi, Modelling and mathematical problems related to tumor evolution and its interaction with the immune system. Mathematical and Computer Modelling, 2000, vol. 32, no. 3-4, p. $413-452$.

[11] N. Bellomo, A. Bellouquid, D. Knopoff, From the microscale to collective crowd dynamics. Multiscale Modeling $\&$ Simulation, 2013, vol. 11, no. 3, p. 943-963.

[12] N. Bellomo, M. Winkler, M. A degenerate chemotaxis system with flux limitation: Maximally extended solutions and absence of gradient blow-up. Communications in Partial Differential Equations, 2017, vol 42, no. 3, p. 436-473.

[13] A. Bellouquid, M. Delitala, Mathematical modeling of complex biological systems, Birkhũser Boston, 2006.

[14] D.P. Bertsekas, Nonlinear programming, Belmont: Athena scientific, 1999, p 1-60. 
[15] M. Bisi, L. Desvillettes, From reactive Boltzmann equations to reaction-diffusion systems, Journal of statistical physics, 2006, vol. 124, no. 2-4, p. 881-912.

[16] P.A. Bliman, N. Vauchelet, Establishing traveling wave in bistable reaction-diffusion system by feedback, IEEE Control Systems Letters, 2017, vol. 1, no 1, p. 62-67.

[17] J.F. Bonnans, J.C. Gilbert, C. Lemaréchal, C.A. Sagastizábal, Numerical optimization: theoretical and practical aspects. Springer Science $\&$ Business Media, 2006.

[18] D. Burini, L. Gibelli, N. Outada, A Kinetic Theory Approach to the Modeling of Complex Living Systems. In Active Particles, Springer International Publishing, 2017, vol. 1, p 229-258.

[19] P. Cannarsa, G. Floridia, A.Y. Khapalov, Multiplicative controllability for semilinear reactiondiffusion equations with finitely many changes of sign, J. Math. Pures Appl., vol. 108, Issue 4, October $2017,425-458$.

[20] J.A. Carrillo, Y.P. Choi, S.P. Perez, A Review on Attractive-Repulsive mean fields for Consensus in Collective behavior. In Active Particles, Springer International Publishing, 2017, vol 1, p. 259-298.

[21] E. Casas, C. Ryll, F. Tröltzsch, Sparse optimal control of the Schlögl and FitzHugh-Nagumo systems, Comput. Methods Appl. Math, 2013, vol. 1, p. 1-29.

[22] F.A. Chalub, P.A. Markowich, B. Perthame, C. Schmeiser, Kinetic models for chemotaxis and their drift-diffusion limits, Monatshefte für Mathematik, 2004, vol. 142, no. 1-2, p. 123-141.

[23] J.-M. Coron, Control and nonlinearity. Mathematical Surveys and Monographs, 136. American Mathematical Society, Providence, RI, 2007. xiv+426

[24] J.-M. Coron, E. Trélat, Global steady-state controllability of one-dimensional semilinear heat equations. SIAM journal on control and optimization, 2004, vol 43, no. 2, p. 549-569.

[25] J.-M. Coron, E. Trélat, Global steady-state stabilization and controllability of 1-D semilinear wave equations, Commun. Contemp. Math., 2006, vol. 8, no. 4, p. 535-567.

[26] E. Cristiani, B. Piccoli, A. Tosin, Multiscale modeling of granular flows with application to crowd dynamics. Multiscale Modeling $\&$ Simulation, 2011, vol. 9, no. 1, p. 155-182.

[27] R. Durrett, Ten lectures on particle systems. Lectures on Probability Theory, 1995, p. 97-201.

[28] R. Durrett, Crabgrass, measles, and gypsy moths: an introduction to modern probability. Bulletin of the American Mathematical Society, vol. 18, no. 2, p. 117-143.

[29] H.W. Engl, M. Hanke, A. Neubauer, Regularization of inverse problems, Springer Science E Business Media, 1996, vol. 375 .

[30] T. Erneux, G. Nicolis, Propagating waves in discrete bistable reaction-diffusion systems. Physica D: Nonlinear Phenomena, 1993, vol. 67, no. 1-3, 237-244.

[31] L.A. Fernández, A.Y. Khapalov, Controllability properties for the one-dimensional Heat equation under multiplicative or nonnegative additive controls with local mobile support. ESAIM: Control, Optimisation and Calculus of Variations, 2012, vol. 4, no. 18, p. 1207-1224.

[32] P.C. Fife, J.B. McLeod, The approach of solutions of nonlinear diffusion equations to travelling front solutions, Archive for Rational Mechanics and Analysis, 1977, vol. 65 no. 4, p. 335-361.

[33] F. Filbet, P. Laurençot, B. Perthame, Derivation of hyperbolic models for chemosensitive movement, Journal of Mathematical Biology, 2005, vol. 50, no. 2, p. 189-207.

[34] R.A. Fisher, The wave of advance of advantageous genes, Annals of eugenics, 1937, vol. 7 no. 4 , p. 355-369.

[35] W.H. Fleming, R.W. Rishel, Deterministic and Stochastic Optimal Control, Springer-Verlag, Berlin, 1975. 
[36] A. Forsgren, P.E. Gill, M.H. Wright, Interior methods for nonlinear optimization, SIAM review, 2002, vol 4, no. 44, p. 525-597.

[37] R. Fourer, D.M. Gay, B.W. Kernighan, AMPL: A mathematical programming language. Murray Hill, 1987, NJ 07974: AT\&T Bell Laboratories.

[38] M. Di Francesco, S. Fagioli, M.D. Rosini, G. Russo, Follow-the-leader approximations of macroscopic models for vehicular and pedestrian flows. In Active Particles, Springer International Publishing, 2017, vol 1, p. 333-378.

[39] F. Hecht, O. Pironneau, A. Le Hyaric, K. Ohtsuka, FreeFem++ Manual, 2005. Website: http://www.freefem.org/

[40] T. Hillen, H.G. Othmer, The diffusion limit of transport equations derived from velocity jump processes, SIAM J. Appl Math. 61, 2000, pp. 751-775.

[41] A.L. Hodgkin, A.F. Huxley, A quantitative description of membrane current and its application to conduction and excitation in nerve, The Journal of physiology, 1952, vol. 4, no. 117, p. 500.

[42] N. Iriberri, J.-R. Uriarte, Minority language and the stability of bilingual equilibria, Rationality and Society, 2012, vol. 24, no. 4, p. 442-462.

[43] Y. Itô, H. Kawamoto, Number of generations necessary to attain eradication of an insect pest with sterile insect release method: A model study, Researches on Population Ecology, 1979, vol. 20, no. 2, pp. 216-226.

[44] A.R. Kanarek, C.T. Webb, Allee effects, adaptive evolution, and invasion success, Evolutionary Applications, 2010, vol. 2, no. 3, p. 122-135.

[45] M. Kàrn'y, Towards fully probabilistic control design, Automatica, 1996, vol. 32, p. 1719-1722.

[46] J.P. Keener, J. Sneyd, Mathematical physiology, New York: Springer, 1998.

[47] W. Klassen, C.F. Curtis, History of the sterile insect technique. In : Sterile Insect Technique, Springer Netherlands, 2005, p. 3-36.

[48] E.F. Knipling, Sterile-male method of population control. Science, 1959, vol. 130, no.3380, pp $902-904$.

[49] J.P. Laplante, T. Erneux, Propagation failure in arrays of coupled bistable chemical reactors. The Journal of Physical Chemistry, 1992, vol. 96, no. 12, p. 4931-4934.

[50] M.A. Lewis, P. Kareiva, Allee dynamics and the spread of invading organisms. Theoretical Population Biology, 1993, vol. 2, no. 43, p. 141-158.

[51] A.M. Liebhold, P.C. Tobin, Population ecology of insect invasions and their management, Annu. Rev. Entomol., 2008, 53, 387-408.

[52] J.-L. Lions, Contrôle optimal de systemes gouvernés par des équations aux dérivées partielles, Dunod, Gauthier-Villars, 1968.

[53] A. De Masi, P. Ferrari, and J. Lebowitz. Reaction diffusion equations for interacting particle systems. J. Stat. Phys. , 1986, vol. 44, no. 589-644, 1986.

[54] J.D. Murray, Mathematical Biology. II Spatial Models and Biomedical Applications, Interdisciplinary Applied Mathematics V. 18, Springer-Verlag New York Incorporated, 2001.

[55] J. Nagumo, S. Yoshizawa, S. Arimoto, Bistable transmission lines, IEEE Transactions on Circuit Theory, 1965, vol. 3, no. 12, p. 400-412.

[56] C. Neuhauser, Mathematical challenges in spatial ecology, Notices of $A M S$ 48, 2002, no. 11, p. 13041314. 
[57] A. Okubo, S.A. Levin, Diffusion and ecological problems, modern perspectives. Interdisciplinary and Applied Mathematics, Springer, 2001.

[58] B. Perthame, Mathematical tools for kinetic equations. Bulletin of the American Mathematical Society, 2004, vol. 41, no. 2, p. 205-244.

[59] B. Perthame, Parabolic equations in biology. In : Parabolic Equations in Biology, Springer International Publishing, 2015. p. 1-21.

[60] D.L. Phillips, A technique for the numerical solution of certain integral equations of the first kind, J. Assoc. Comput. Mach., 9 (1962), pp. 84-97

[61] J.-P. Raymond, H. Zidani, Hamiltonian Pontryagin's principles for control problems governed by semilinear parabolic equations, Applied Math. Optimization, 1999, vol. 2, no. 39, p. 143-177.

[62] A.P. Ruszczyński, Nonlinear optimization, Princeton university press, 2006, vol. 13.

[63] C. Ryll, J. Löber, S. Martens, H. Engel, F. Tröltzsch, Analytical, optimal, and sparse optimal control of traveling wave solutions to reaction-diffusion systems. In Control of Self-Organizing Nonlinear Systems, Springer International Publishing, 2016, pp. 189-210.

[64] A. Stevens, The derivation of chemotaxis equations as limit dynamics of moderately interacting stochastic many-particle systems, SIAM J. Applied Math., 2000, vol. 61, no. 1, p. 183-212.

[65] C. Ryll, J. Löber, S. Martens, H. Engel, F. Tröltzsch, Analytical, Optimal, and Sparse Optimal Control of Traveling Wave Solutions to Reaction-Diffusion Systems, In : Control of Self-Organizing Nonlinear Systems, Springer International Publishing, 2016, p. 189-210.

[66] J.C. Strikwerda, Finite difference schemes and partial differential equations. Society for Industrial and Applied Mathematics, 2004.

[67] M. Strugarek, N. Vauchelet, Reduction to a Single Closed Equation for 2-by-2 Reaction-Diffusion Systems of Lotka-Volterra Type, SIAM J. Applied Math., 2016, vol. 76, no 5, p. 2060-2080.

[68] C.R. Taylor, Determining optimal sterile male release strategies, Environmental Entomology, 1976, vol. 5 , no. 1 , pp. 87-95.

[69] A.L. Tits, A. Wächter, S. Bakhtiari, T.J. Urban, C.T. Lawrence, A primal-dual interior-point method for nonlinear programming with strong global and local convergence properties, SIAM J. Optim., 2003, vol. 1, no. 14, p. 173-199.

[70] F. Tröltzsch, Optimal control of partial differential equations, Graduate studies in mathematics, 2010.

[71] A. Wächter, An Interior Point Algorithm for Large-Scale Nonlinear Optimization with Applications in Process Engineering, PhD Thesis, Carnegie Mellon University, 2002.

[72] M. Winkler, N. Bellomo, Finite-time blow-up in a degenerate chemotaxis system with flux limitation, Transactions of the American mathematical society, Series B, vol. 4, p. 31-67, 2017

[73] B. Ycart, Modèles et algorithmes markoviens, Springer Science ES Business Media, vol. 39, 2002.

[74] E. Zuazua, Controllability and Observability of Partial Differential Equations: Some results and open problems, in Handbook of Differential Equations: Evolutionary Equations, vol. 3, C.M. Dafermos and E. Feireisl eds., Elsevier Science, 2006, pp. 527-621. 